\title{
La enseñanza de enfermería, un análisis desde las teorías de la reproducción
}

\section{Nursing teaching, an Analysis from the Reproductionist Theories Perspective}

Lic. Beatriz Carmona Mejía•

Mtra. Gandhy Ponce Gómez••

\section{Resumen}

Con un creciente conocimiento que identifica nuestras tareas sociales y de grupo, a través de esfuerzos compartidos para mejorar el futuro de nuestra calidad de vida, la enfermería se encuentra en constante evolución. Estas preocupaciones no son nuevas; la práctica de la enfermería, históricamente, ha estado desarrollando una base científica, que de acuerdo con postulados positivistas, le da sentido a la disciplina. En este proceso, la educación de la enfermería ha estado influida por diversas entidades con ideología capitalista tales como: religión, escuela, familia, leyes, política, sindicatos, los medios, y la cultura. En este artículo, se describen algunos postulados reproduccionistas, que de acuerdo con las perspectivas filosóficas de Carlos Marx, Louis Althusser, Baudelot y Establet, Boules y Gintis; Bordieu y Passeron entre otros, pueden influir en la educación de enfermería contemporánea.

\section{Abstract}

With a growing knowledge which identifies our social and group tasks, and by sharing efforts to improve the future of our quality of life, nursing is in a constant process of evolution. These concerns are not new; nursing practice has been historically developing a scientific base, which according to positivist postulates, gives sense to the discipline. In this process, nursing education has been influenced by diverse capitalistic-ideology entities such as: religion, school, family, law, politics, work unions, media, and culture. In this study, described some Reproductionist postulates, which according to the philosophic perspectives of Carlos Marx, Louis Althusser, Baudelot and Establet, Boules and Gintis; Bordieu and Passeron, among others, can be as the contemporary education of nursing.
Palabras

Clave:

Teorías de la

reproducción, enfermería, disciplina.

\section{Key} Words:

Reproductionist

theories, nursing

discipline.

-Profesor de Carrera Asoc. C. FES ZARAGOZA. UNAM.

..Profesor de Carrera Asoc. B. Unidad de Investigación. ENEO- UNAM.

RECIBIDO 8 ENERO 2011

ENVIADO 22 FEBRERO 2011

ACEPTADO 26 DE ABRIL 2011 


\section{Contextualización}

Actualmente la enfermería se encuentra en proceso de construcción, clarificación y establecimiento de su naturaleza como disciplina profesional, preocupándose además por crear un cuerpo de conocimiento que identifique gremial y socialmente su quehacer y dominio.

En el proceso de edificación disciplinar de enfermería se reconoce igual que otras disciplinas el que han tenido que atender a un sistema de formación educativo propio de "sociedades de tipo capitalista" mismo que ejerce su función dominadora a través de aparatos ideológicos religiosos, escolar, familiar, jurídico, político, sindical, informativo, y cultural. ${ }^{1}$

De acuerdo con las Teorías de reproducción, la intención básica de los procesos de enseñanza aprendizaje en los colegios e institutos es preparar estudiantes para un desempeño eficiente con un perfil trabajador, adaptable, obediente y cooperador e integrado a la estructura jerárquica dentro de un modelo de relaciones laborales típico de la economía capitalista. Y es a partir de los supuestos de esta corriente educativa que se da inicio al análisis de la evolución histórica de la disciplina de enfermería, que irán siendo comparadas con los puntos de vista de los sociólogos y economistas de las teorías de la reproducción social.

El análisis de la enseñanza de la enfermería se inicia con el concepto griego de Razón, denominada así debido al desarrollo intelectual del ser humano, cuyos representantes más destacados se ubican en Grecia la cual gozaba de prestigio intelectual y artístico con figuras como Aristóteles, a quien su insa- ciable conocimiento del proceso de la vida le condujo a disecar animales sentando así la base para la biología y el estudio de la anatomía comparada; Hipócrates establece los fundamentos de la medicina moderna; y Sócrates y Platón dan fundamento a la filosofía y el gobierno político. La influencia de la cultura griega en el mundo provocará un cambio de los métodos de pensar y observar la realidad, en el caso de la salud sentó las bases para dilucidar la diferencia entre lo sano y lo enfermo. ${ }^{2}$

El surgimiento de la Enfermería Moderna en el siglo XIX, se documenta a partir del proyecto de Florence Nightingale, quién desde su juventud decidiría ser enfermera a causa de las deficientes condiciones que tenía este oficio en los hospitales de Inglaterra. ${ }^{2}$ En 1860 , Nightingale empezó la reforma de enfermería mediante la creación de una escuela modelo en la que se iba a enseñar el arte de la enfermería.

En la escuela, dirigida por Nightingale ubicada en el Hospital de Santo Tomás, en Londres, en conexión estrecha con una escuela de medicina, rica e influyente, dio inicio un proyecto educativo de formación de estudiantes de enfermería (residentes en el hospital) que obedecía a la necesidad imperiosa de mano de obra que se hiciera cargo del cuidado diario y continuo de pacientes, ancianos, leprosos, etc. ${ }^{3}$ En este contexto eran los médicos quienes tomaban la temperatura, aplicaban inyecciones hipodérmicas y en ocasiones cambiaban las sábanas de los pacientes gravemente enfermos. Es aquí donde se comienza a visualizar la formación de alumnos para trabajo diario con técnicas eficientes, orientadas ex- clusivamente a ejecución de actividades manuales o para las que apenas se precisaba reflexión.

A la luz de los postulados de Marx quien justificaba que en las escuelas se exageraba en la visión de vincular los conocimientos adquiridos con el mundo de producción; ${ }^{4}$ se explica así como las estudiantes de enfermería de Nightingale y de hoy además de recibir instrucción, habían de estar en total contacto con la experiencia laboral a la que habrían de insertarse. Según el orden laboral de la época marxista, los inspectores de fábricas descubrieron rápidamente que los niños y adolescentes que seguían este régimen de enseñanza analizado por Marx, ${ }^{5}$ aprendían tanto y a veces más, que los alumnos de escuelas corrientes: El sistema de mitad trabajo y mitad escuela une a la enseñanza con la producción; este hecho ya prevalecía desde fines del siglo XVIII.

La reglamentación de la vida en el hogar - escuela propuesto por F. Nightingale correspondió inevitablemente a la era victoriana, en la que a pesar de que las mujeres empezaban a aventurarse fuera del amparo de la vida familiar, una estudiante no podía salir sola, sino que era obligatorio que otra estudiante le hiciera compañía. En la institución citada un cura pronunciaba dos veces a la semana un sermón; y todo acto era vigilado por la Matrona Sarah Elizabeth Wardroper, directora de la escuela Nightingale, de forma que no hubiera dudas acerca del comportamiento o el empleo indebido de la libertad no acostumbrada para las mujeres. Bajo la óptica de Althusser, citado por Gil, se postula la existencia de los Aparatos Ideológicos del Estado 
(fuerzas de dominio y control sobre los individuos); que funcionan masivamente y mediante la ideología, pero secundariamente funcionan a través de la represión; ${ }^{1}$ que para el caso de la escuela de enfermería se traduce en normas rígidas de comportamiento y sobre todo para la mujer; y por otro, el control por medio de las ideas religiosas a las que se veían sometidas las estudiantes.

Dentro de la estructura citada es evidente que estaban representados entre las mujeres de la escuela dos grados sociales, como lo habían estado en el grupo de enfermería de la Guerra de Crimea, a saber: aspirantes instruidas de la clase superior, que pagaban unas treinta libras anuales en concepto de colegiatura, y un grupo que no pagaba, seleccionado por su carácter y condiciones económicas, se trataba de una capa menos distinguida de la sociedad; incluso eran las de clase alta quienes al final de la instrucción de cuatro años eran las privilegiadas para ocupar también puestos de matronas o superintendentes. Haciendo la comparación con los supuestos de Marx ${ }^{6}$ citado en Giroux, esto reflejaba los niveles por los que estaba constituida toda sociedad, las clases ricas o capitalistas y las pobres o laborales; aunado a las ideas de Baudelot y Establet $^{6}$ que visualizaron la función de la escuela en la división de la sociedad en dos clases antagónicas y la dominación de la burguesía sobre el proletariado, ${ }^{6}$ que estaba enmascarada al aceptar supuestamente a las clases ricas, lo mismo que a las pobres ${ }^{1}$. Además en las ideas también de Baudelot y Establet, a los futuros proletarios se les impartía un cuerpo compacto de ideas burguesas simples; mientras que a los futuros burgueses aprendían y aprenden a convertirse en intérpretes, en actores e improvisadores de la ideología burguesa, de esta parte es obvio que estaban encargadas las Matronas o Superintendentes, antes estudiantes de clase alta con el afán de vivir la experiencia de la enfermería.

Por otra parte, los esfuerzos y el sacrificio por el abandono de la vida hogareña para las estudiantes sólo de clase alta, fue recompensado con esos puestos de jerarquía que eran de instructoras o matronas, y de acuerdo con Bowles y Gintis, estas etiquetas se otorgan a los individuos con características personales que son relevantes para la distribución de tales puestos (autosuficiencia, liderazgo, independencia). ${ }^{7}$

La necesidad urgente de escuelas de enfermería del tipo de la famosa escuela Nightingale, se extendió a toda Inglaterra, Europa y Norteamérica, por desgracia en el afán de ser "eficientes" en egresar enfermeras, la imitación se apoderó de la supuesta capacitación en estas escuelas, dejando de lado por desgracia la instrucción clínica junto a la cama del paciente como lo proponía Nightingale, incluso a falta de expertas que dieran instrucción a las aprendices, a partir del segundo año de estudio ellas mismas se hicieron cargo de otras responsabilidades más que nada manuales para dar cuidado a los enfermos al mayoreo, y de una forma aún más irreflexiva.

Según la postura de Baudelot y Establet, es inevitable, que surjan determinadas resistencias resultado del instinto de clase o conciencia espontánea de la explotación, ejem- plificado en los primeros días de las escuelas profesionales de enfermería, en que se dio el auge de críticas por la falta de instrucción teórica en mujeres inteligentes inquietas como lo fueron Isabel Hampton Robb o Lavinia L. Dock, que manifestaban una falta de satisfacción intelectual en la presurosa práctica de enfermería que se les imponía. ${ }^{3}$ Comparando con los postulados reproduccionistas se visualiza que la ideología proletaria no está constituida por ideas o por conceptos, es decir que las enfermeras poco a poco se dieron cuenta de que atendían a una filosofía utilitarista, por el hecho de ser "hacedoras," con escaza e involuntaria formación técnica.

Con respecto a la currícula en las escuelas de enfermería fuera de Inglaterra, Juana Hernández Conesa describe como los primeros libros de texto de enfermería éstos fueron Manuales (la escuela profesional de Connecticut, publicó el primero en 1878) y libros de medicina, pero no aquellos libros con los que en su época se preparaba a los médicos, sino aquellos que eran donados a las escuelas de enfermería ya que medicina ya los consideraba obsoletos. Hablando de la contemporaneidad, sigue siendo una práctica común en algunas escuelas de enfermería, el e studiante investigue en libros que tiene a la mano con algunos años de atraso.

La labor de la clase en las escuelas de enfermería del siglo XIX se centraba alrededor de la práctica, ética, anatomía, fisiología, materia médica e higiene. Los docentes predominantemente eran médicos, encargados de estructurar los programas. Impartían nociones básicas no siempre sistematizadas, ya que concebían a la enfermería 
en una dimensión exclusivamente técnica. Las aspirantes a preparación profesional habían de reunir cierta cultura, juventud (25 a 35 años) y una constitución sana. Los requisitos culturales se concebían similares a los otorgados en escuelas tradicionales o conventos, aunados a una educación de principios y moralidad que la madre en su casa culminaba. ${ }^{8}$ Incluso las escuelas resultaron tan atractivas que hasta mujeres de clases superiores, dejaban sus casas para atender a los enfermos. Ya para las dos últimas décadas del siglo XIX, las estudiantes eran admitidas a las escuelas no por clases o por grupos, sino como iban egresando las generaciones anteriores, en donde los cursos iniciaron de un año, luego dos, hasta llegar a tres años de preparación.

En el siglo XIX era común el alojamiento en residencias para estudiantes; consolidándose el principio de que la enfermera atendiera a su paciente con abnegación; "cuanto más agotada se le viera en su aspecto exterior, y cuanto mejor pudiera soportar la incomodidad y la fatiga personales, tanto más se acercaba al ideal de la buena enfermera". En la comparación con las teorías de la reproducción, las escuelas eran consideradas espacios en los que se aprende tanto una serie de habilidades como las reglas del buen comportamiento, que en palabras de Althusser: "la reproducción de la fuerza de trabajo exige, no solo una reproducción de su cualificación, sino también, y simultáneamente, una reproducción de su sumisión a las reglas del orden establecido; a la ideología dominante por parte de los obreros en su capacidad de asimilar la ideología de la explotación y de la represión, a fin de que se aseguren también "mediante la palabra" el dominio de la clase dominante. ${ }^{1}$

En enfermería, esto era evidente al hacer hincapié en las actitudes de sumisión, bondad, servilismo y abnegación con la que se debía contar para ser enfermera, la enfermera hasta la fecha tiene construida su propia imagen con estas características y muchas la siguen asumiendo, existen aún ahora escuelas con estos patrones de enseñanza.

Además en comparación con los postulados de la teoría de la Correspondencia, se empezó a notar la diferencia de que no importaban demasiado los contenidos de la enseñanza, o de los difundidos en los textos, sino que estaban adquiriendo especial relevancia las experiencias cotidianas a través de las estrategias de enseñanza - aprendizaje que se vivían y aún continúan viviendo en aulas (el currículo oculto). Bowles y Gintis, plantearon

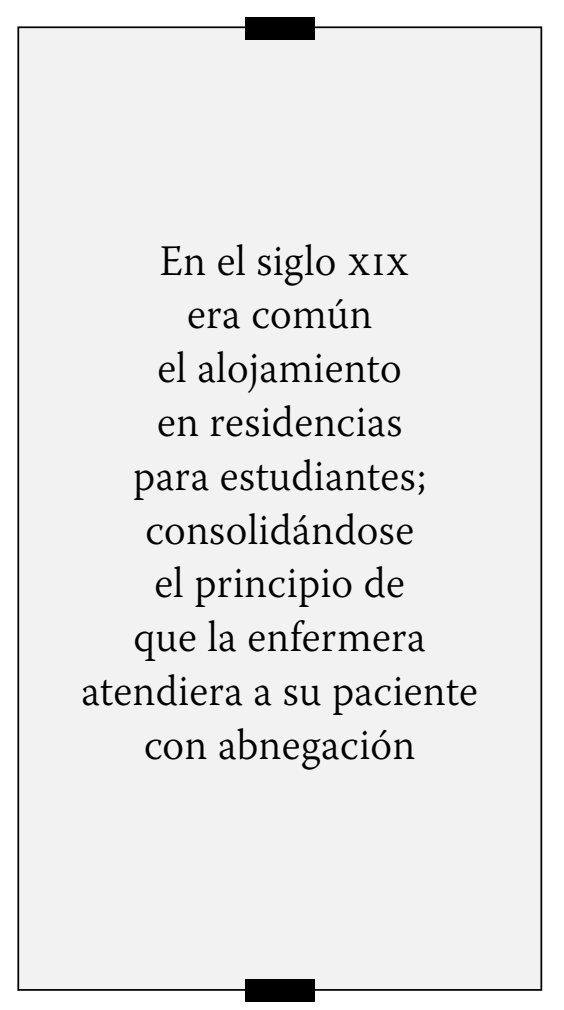

que las capacidades cognitivas y afectivas desarrolladas, como las destrezas técnicas contribuyen a la construcción de imágenes de uno mismo, que implícitamente van a colaborar para legitimar y darle un sentido de obviedad o naturalidad a la existencia de desigualdades sociales y económicas. ${ }^{7}$

Referente a la adopción de uniformes, en 1870 se comenzó a instituir el uso de éste, tanto por consideraciones de economía y limpieza, como por generar en el grupo de enfermeras una conciencia moral aumentada y al abandono de la vida personal, dentro de los hospitales; y en 1875, se difundió el uso de algún tipo de tocado, con el fin de cubrir el pelo por higiene. En particular el origen de la cofia se remonta a los días de la edad media e inclusive de tiempos anteriores en los que la humildad de la mujer se manifestaba por la asunción de un velo nupcial. ${ }^{3}$ El gorro de la enfermera era el símbolo de su servicio a favor de la humanidad. Ya fue muchos años después que se le asignó la función higiénica de cubrir el cabello o los peinados altos de mujeres cuyo cabello llegaba más debajo de la cintura. En general al surgir los primeros uniformes, estos fueron tomando diversidad convirtiéndose en distintivos para cada escuela en particular. Pero esto en el análisis reproduccionista de Althusser es una ideología que fue poco a poco permeada en las escuelas, estableciendo ritos, rutinas y prácticas sociales, que mucho se dejó ver en la forma de adoptar el uniforme, precisamente por el significado que la misma palabra abarca, un uniforme como vestimenta única, formal y reglamentaria, que implica que el sujeto es parte de un grupo y que 
reconoce por tanto la autoridad de éste sobre él.

Por otra parte, las afirmaciones teóricas de Bordieu* donde se explica el proceso de dominación donde éste está frecuentemente forjado, a través de una correlación entre una cierta disposición (habitus*) y las expectativas e intereses incluidos en la posición de instituciones específicas (habitat). ${ }^{9}$ Entonces, en enfermería, esta relación se devela al aceptar el uso de uniformes, tocados, peinados y el abandono de la vida personal (habitus para la enfermería) con su aceptación por parte del habitat de esa época, es decir, los hospitales - escuela.

La necesidad de una acción concertada entre las enfermeras se hizo sentir primero en Inglaterra. Mientras que en Norteamérica, se dio relevancia a generar más escuelas con el modelo Nightingale, lo que respondía a las propuestas eficientistas de las teorías tylerianas. En 1887, en Inglaterra se constituyó la asociación de enfermas graduadas en la Royal British Nurse's Association, cuyo objetivo era el de reunir a todas las enfermeras graduadas para la protección de sus intereses comunes, promover las medidas para la mejora de la preparación profesional $y$, finalmente, conseguir el registro oficial de todas las graduadas de las escuelas de enfermería como profesionales instruidas y competentes, con una preparación concreta y oficialmente reconocida. ${ }^{3}$ Pero para ese entonces este esfuerzo no dio frutos, ya que hubo quien se opuso a reglamentar la enfermería, ya que era considerada una vocación, y dentro de este grupo de oposición se encontraba Nightingale por considerarlos actos po- líticos que lo único que lograrían sería minimizar la vocación que ella consideraba indispensable para esta profesión. Incluso es Antonio Gramsci, ${ }^{10}$ estableciendo la comparación con las teorías reproduccionistas quien manifiesta que el Estado en pro de favorecer las condiciones de acumulación de capital, interviene al requerir a los trabajadores o profesionales los certificados establecidos por él mismo, los sistemas educacionales, ya desde el siglo XIX se inclinaban con gran peso hacia una producción educativa altamente tecnocrática. Pero además el efecto capitalista es más visible en la distinción que se hace a todo nivel entre el conocimiento de alto status de las ciencias biológicas y matemáticas, y el conocimiento de bajo status de las humanidades, que es el caso de la enfermería.

Pero los esfuerzos de grupos hegemónicos, no pararon ante un pequeño obstáculo, y poco a poco fueron surgiendo asociaciones de enfermería. Y ya para 1899 , se tenía la propuesta formal para crear el Consejo Internacional de Enfermeras (CIE), con el propósito declarado de establecer y mantener normas elevadas de servicio de enfermería, de la enseñanza de la enfermería y de la ética profesional en todo el mundo. El CIE, hoy en día tiene más fuerza que nun$\mathrm{ca}$, e incluso otras asociaciones o grupos colegiados buscan elevar su reputación asociándose a éste o a la American Nurse's Association (ANA). También se reconoce la fuerza de asociaciones, por ejemplo en México de la Federación Mexicana de Asociaciones de Facultades y Escuelas de Enfermería A.C. (FEMAFEE), el Colegio Nacional de Enfermeras o la Asociación Latinoamericana de Escuelas de Enfermería, etc.

A partir de estos esfuerzos de congregación, surgen otros, que desean saber las formas en que se lleva a cabo la formación de enfermeras, es así como la Asociación Rockefeller en 1923 , financió una serie de estudios en el campo entero de la enfermería, cuyos hallazgos fueron 1) muchas enfermeras en puestos administrativos, con escasa preparación, 2) escasas enfermeras otorgando atención domiciliaria, 3) carencia de enfermeras con conocimientos en salud pública, 4) escuelas con materiales y laboratorios demasiado rústicos, 5) predominancia de los postulados biomédicos en la formación de enfermeras; entre otros. ${ }^{2}$

Es ya para los 50 s que se da auge a la necesidad de reestructurar los programas de formación de enfermería también a raíz de los trabajos de Ralph Tyler e Hilda Taba con su obra "Principios básicos del currículo" que influyó poderosamente en los educadores norteamerica-

\footnotetext{
*Bordieu, define al habitus como un sistema de esquemas de percepción, pensamiento y acción adquiridos durablemente, engendrado por condiciones objetivas pero tendiente a persistir aún después de una alteración de aquellas condiciones. El hábitus es un producto de la socialización y la historia corporeizada, y difiere entre los variados grupos subordinantes y dominados dentro de la sociedad.
} 
nos y latinoamericanos. ${ }^{11}$ De ahí se observa la necesidad de que exista una coherencia interna en los planes de estudios de enfermería, pero que además existiera la flexibilidad para compartir cursos, seminarios y demás actividades académicas, renovar las modalidades pedagógicas, ya que a pesar de haber dejado décadas atrás la "corriente educativa tradicional", aun seguía y sigue practicándose el magistrocentrismo, y además de poner énfasis en la enseñanza de distintas lenguas independiente de su vocablo natal; la necesidad de incluir formación en salud pública, geriatría, administración de servicios de salud, psiquiatría, etc. Aún nos encontramos en la lucha por conformar el cuerpo de conocimientos de la disciplina, dilucidar al cuidado como objeto de estudio, fortalecer un lenguaje común a nivel internacional, promover las redes de comunicación y por tanto la divulgación de los avances en investigación, docencia, administración y cuidado en sus distintos escenarios.

Los planes de estudios de las escuelas de enfermería, en particular de la licenciatura norman la preparación de 4 años y el riguroso año de servicio social, que en realidad sigue predominando en las zonas urbanas y en unidades hospitalarias. Es decir se sigue sin responder en realidad a las necesidades sociales planteadas para la enfermería como una práctica social.

Finalmente en este siglo XXI se observa que el paradigma biomédico en la profesión de enfermería aun subsiste, ya sea en planes de estudios tradicionales, o en planes de estudio ya reestructurados. La enfermería de hoy obedece a los criterios que movieron a las pri- meras enfermeras, en su idea de insertarse en el medio laboral; avalando más que nunca la corriente reproduccionista, impuesta desde los aparatos ideológicos del estado mismo, "convenciendo" a los futuros profesionales y los ya trabajadores de la salud, haciéndoles ver que el obtener una plaza en el Sistema de salud son logros, casi extraordinarios, con los que deben de estar felices.

\section{Conclusiones}

Hoy en día en las escuelas de enfermería, como en el resto de escuelas de educación superior y en consecuencia a las sugerencias de movimientos económicos - políticos internacionales (el Tratado de Libre Comercio de América del Norte y la Globalización), ya viven la formación de profesionales que se circunscriben al ámbito de la producción, y no al de las necesidades sociales. Se promueve la competencia entre sujetos, programas e instituciones, supuestamente para acreditar los programas y certificar a los profesionales, pero que como dice Bowles y Gintis, se vuelve destructiva.

El conocimiento adquiere un valor práctico, fenómeno visualizado ya por Carlos Marx; domina el carácter tecnologizante y se minimizan los proyectos de investigación y docencia en las áreas humanísticas, limitante de la cual adolece la enfermería. En correspondencia con la teoría del capital humano, se pone énfasis en la rentabilidad individual y social de la educación, y se sobrepone el valor del mercado a otros valores de la formación universitaria. Se propone mejorar la selección de profesores, evaluarlos y pagarles según

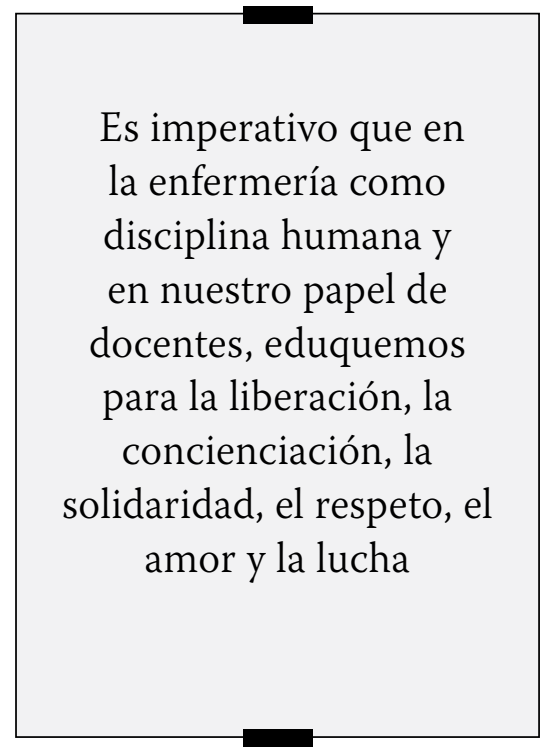

el mérito, recordemos el término meritocracia, donde se ponen los obstáculos y las brechas delgadas y endebles para permitir que sólo pocos de los no privilegiados (clase trabajadora) puedan acceder a este tipo de mejoras salariales y "a una vida mejor y feliz"?

La preocupación por la calidad que existe en la formación de profesionales de enfermería, en realidad obedece a la necesidad de producción de mano de obra más barata, sumisa, obediente y adaptable al status quo. Lo que en realidad acontece es la diseminación del "habitus" como un virus altamente infeccioso y que en lugar de promover valores de libertad, sensibilidad, respeto, lucha y fortaleza interior, sólo ha conducido a la obediencia ciega de la humanidad para sostener al Estado en las distintas formas que ha adoptado, coercitivas, tolerantes y hasta paternalistas, pero cuyo único fin es preservar a los grupos dominantes. ${ }^{12}$

El análisis de la evolución de enfermería bajo el discurso de las Corrientes Educativas repro- 


\section{Enfermería Universitaria}

duccionistas, generan una amplia gama de controversias a las que hemos estado alineados en la escuela (además de la familia, punto que no fue tocado, sin embargo que también es reconocido de gran influencia para la reproducción social). El particular reconocimiento de estos esquemas rígidos a los que se ha sometido al ser humano pero a la que no debemos acostumbrarnos, y si tratar de cambiar primero en lo individual y luego colectivamente.

El futuro no está predeterminado, depende de las opciones de civilización que se hagan y las transformaciones en la educación que se realicen. Los nuevos sistemas educativos deben promover el oponerse a la ceguera del pensamiento simplificador, disciplinario y disciplinado; una educación que haga consciente, reflexivo y propositivo al individuo ante la compleja realidad.

Es imperativo que en la enfermería como disciplina humana y en nuestro papel de docentes, eduquemos para la liberación, la concienciación, la solidaridad, el respeto, el amor y la lucha por saber más allá de lo que se plantea en aula, en la familia e incluso en la sociedad.

\section{Referencias Bibliograficas}

1 Gil R. La importancia de la educación en la determinación de la Hegemonía. Las teorías de la Reproducción. (2004). Disponible en: http://bit.ly/tgIbaa [Consulta $1 \mathrm{de}$ marzo de 2010].

2 Jamieson M, Sewal M, \& Suhrie E. Historia de la Enfermería. Traducido por Gerhard O. México: Interamericana 1968, p 34.

3 Marriner T. Modelos y Teorías en Enfermería. España: Elsevier 2007, p. 132.

4 González R. Teorías sociológicas de la educación, 2008. Disponible en: http://bit.ly/vuWYv8 [Consulta 1 de marzo del 2010].

5 Marx C \& Engels F. Manifiesto del Partido Comunista. Beijing, China: Ediciones en Lenguas Extranjeras 1848, p.167.
6 Giroux H. Teorías de la reproducción y la resistencia en la Nueva sociología de la educación: Un análisis crítico, México, D. F.: Era, Cuadernos Políticos 1985; 44: 36-65.

7 Bowles S, Gintis H. La instrucción escolar en la América capitalista. Madrid: Siglo XXI 1985. p.311.

8 Hernández CJ. Historia de la Enfermería. Un análisis histórico de los cuidados de Enfermería. España: Interamericana McGraw Hill 2005. p.259.

9 Boudieu P y Passeron JC. La reproducción elementos para una teoría del sistema de enseñanza. Barcelona: Laia 1977. p.145

10 Gramsci A. Antología. Madrid: Siglo XXI 1974. p. 129

11 Taba H, Tyler R. Elaboración del curriculum. Buenos Aires: Troquel 1974. p.32.

12 González R. Teorías sociológicas de la educación. (1998). Disponible en: http://bit.ly/rZFCbv [Consulta 1 de marzo del 2004] 